also phthisical, and that is, until he was obviously wasting away. 'This gloomy view of the disease often causes the physician to be chary of investigating for fear of declaring this unpleasant fact, when the patient will resent the suggestion of his having tuberculosis. Moreover, the patient often dreads going to a doctor who he believes will give an honest opinion.

The old myth that pulmonary tuberculosis was almost invariably hereditary has caused many a well-developed man with a good family history to allow his disease to become incurable because he did not know his danger. Again, patients so commonly prefer to frequently spond a little time and money with their physician and be tinkered up, rather than to submit to a thorough overhauling and perhaps a radical treatment at a greater though less frequent expenditure of time and money. Again, the physician who urges a thorough inquiry is often suspected of making too much of a case for selfish ends, and magnifying his own importance by unnecess:ırily alarming his patient.

A verdict of tuberculosis is supposed to always mean exile from home to a more favorable climate. While this is most usually the surest and quickest road toward recovery, there are often individual reasons why it is not the best course to pursue. Sometimes even a slight change of air, and for a short period, is sufficient, if the patient is radically treated as regards the hygiene of his horne and person. At all events, in the very early stages, wliere the progress of the disease is slow, this can be tried.

I find that the poverty of the patient has some ir. fluence on the delay of diagnosis; in those in whom there was no delay, 16 per cent. were poor; in those in whom there was delay, 26 per cent. were poor. Again, showing the intluence of the knowledge of medicine on the patient or his friends; in those in whom there was no delay, 22 per cent. were physicians or relations of physicians; while in those in whom there was delay, there only 9 per cent. were physicians, or of physicians' families.

If ever a suit for malpractice is justified against an educated and honest physician, it certainly is in many of the instances of neglect of the early diagnosis of pulmonary tuberculosis.

Let us remember that under appropriate treatment the large majority of cases of incipient pulmonary tuberculosis get well and remain well if they are instructel in and practice personal hygiene.

\section{CONVULSIONS IN INFANTS AND YOUNG CHILIDREN.}

THEIR POINT OF ORIGIN, NATURE, CADSE AND MANAGEMENT.*

BY C. G. SI.AGLE, M.D. MINNEAPOLIS, MINA.

No apology is demanded for discussing here what is only a symptom and not a disease per se; for of the many sudden, violent, and dangerous affections of very early life, I know of none which demand more prompt, careful, and skilful effort of the physician, than convulsions. Their suddenness, gravity, and frequency give to them a peculiar importance; and it is here, in a peculiar manner, that human life often hangs suspended on a prompt impulse of the physician's trained and cool intelligence. For what can be more terrifying to the parents and friends, or more dangerous often to the little

* Presented to the Section on Diseases of Children, at the Fiftieth Annual Meeting of the American Medical Association, held at Columbus, Ohio, June 6-9, 1899 patient, than an infant in the agonies of a well-developed eclampsia.

Most authors attempt to treat this intricate subject under four headings, or divisions, as: 1. Points of Origin. 2. Nature. 3. Causes. 4. Treatment or Management. But these divisions can not be discussed entirely separately, for in discussing the point of origin, we are necessarily contemplating, to a great extent, the cause, at least the "proximate cause," and also to a considerable extent, the essential nature of convulsions. Nevertheless, as we must systematize what we have to say on the subject, perhaps we can not do better than to follow these divisions as far as possible.

For purpose of study and treatment, convulsions have been variously classified, as to their points of origin, and exciting causes. The late Prof. J. Lewis Smith discussed them under the headings of "Idiopathic," "Symptomatic," and "Sympathetic," the first class being those purely functional-as from sudden, violent emotions, etc.; the second class, those from brain lesions; the third, those occurring as complications of diseases other than the brain-as pertussis, exanthems, etc. Professor Holt groups them under three headings, as those occurring from direct irritation of the cortex of the brain; those from reflex irritation; those from "toxic" influences. Other divisions, as you know, are into "Central" and "Peripheral," or "Centric" and "Excentric," etc. Professor Rotch has tabulated as to causes in an admirable manner under the two significant headings of "Central" and "Peripheral"-or reflex-and I think all of the exciting causes can well be grouped in his classifications.

Morris J. Lewis ${ }^{1}$, for the purpose of differential diag. nosis, etc., groups convulsions into eight classes, as follows:

1. Convulsions may be evidence of reflex irritation from some temporary and comparatively trivial disorder, such as constipation, overloaded stomach, irritation of teething, earache, worms, genital irritation, etc.

2. They may constitute an initial symptom of one of the exanthemata; or some other acute disease, either thoracic, spinal or cerebral, or they may be due to thermic fever.

3. They may indicate that the child is suffering from some general condition of ill health, such as indigestion, rickets, cyanosis, etc.

4. They may indicate gross intracranial disease of more or less chronic nature.

5. They may be a symptom arising during the course of one of the acute diseases, such as whooping-cough, or may be an evidence of toxemia in the exanthemata or kidney disease.

6 . They may be the result of various traumatisms, such as injuries to the head, or a violent hemorrhage; other than cerebral; causing cerebral anemia, or from severe burns or shocks.

7. They may be one of a series arising without assignable cause; and constitute epilepsy.

8. They may indicate nothing more than a "A Mode of Dying," especially in those diseases which are accompanied by severe watery diarrhea and vomiting, as cholera infantum, etc.

This comprehensive summary would seem to embrace most of the exciting causes fairly well grouped; and as Lewis remarks, "Two or more of these classes may be found associated in the same case."

Some such grouping, together with the systematic tabvlation of the various exciting causes, as Dr. Rotch has given us in his lectures, under the headings of "Central" 
and "Peripheral," will do much to facilitate the diagnosis and management of these often intricate symptomatic affections.

I take it for granted that this discussion is understood to exclude the somewhat similar, and often nearly allied, spasmodic neuroses, as puerperal eclampsia, epilepsy, chorea, hysteria, tetany, trismus; and, to some extent, that local spasmodic affection of infants, "laryngospasm." I accordingly confine it for the most part to "an acute affection of infants and young children," arising often without perceptible structural lesions of the nervous systems and characterized by partial or general convulsions, either "tonic" or "clonic," accompanied by more or less complete loss of consciousness. In other words, I limit it, as far as possible, to "idiopathic general convulsions of young children."

Points of Origin.-Many diligent and ingenious investigations as to the points of origin and "proximate cause" of convulsions have been made by such distinguished investigators as Kussmaul, Tenner, BrownSéquard, Nothnagle, Gowers, Dickson, Marshall Hall, J. Hulings Jackson, Allbutt, Jacobi, Hammond, and a host of others, both in this country and abroad; but up to the present, without satisfactory or demonstrable conclusions; or even anything like uniformity in the theories deduced from their painstaking experiments and observations, and this part of our subject seems to be yet sublite. I do not therefore believe this is the proper place to go into a discussion in detail, of the various theories deduced from experimentations which have been presented from time to time as to the exact origin, or proximate cause and essential nature of these convulsions; they are too many and various and contradictory to be profitably discussed in a limited paper.

That much is yet to be learned in this direction seems certain. Holt says: "They are motor discharges from the cortex of the brain." Morris J. Lewis says: "Convulsions are in all probability due to an exaltation of the lower nerve-centers; or more frequently, to a suspension of the inhibitory power of the higher cerebral centers"-or both of these conditions may exist at the same time-and further, "It remains to be said that we are still very much in the dark as to the immediate processes producing convulsions."

Taylor and Wells" have well said: "Infants have their nervous system in process of rapid development-only the component but undifferentiated parts of which are in great activity, ready to receive and re-energize limitless new impressions." This may be done systematically and wholesomely, or irregularly and excessively, resulting in growth and development or explosively doing harm. At birth, the lower centers only are developed, hence control is limited until the higher centers become competent to exert inhibition; hence in the earlier months of life convulsions are common, and less so after two years.

Peterson ${ }^{3}$ says: "Convulsions, whether local or general, have their origin in katobolic discharges of nerve cells, either in the cortex, or at the base of the brain." Further, he remarks: "There is no form of eclampsia gericrated from the ganglion cells in the spinal cord." This rcmark is made after reviewing and dissenting from J. Hulings Jackson's theory, that there are three levels from which such discharges may occur-as from the cells of the pontobulbar region, from the Rolandic area, and from a level which he (Jackson) conceives to exist in the frontal lobes, and to represent the highest control of sensory-motor functions. However, after quoting these plausible views without further comment, and having nothing new to offer or suggest on this intricate part of our subject, I shall pass at once to the consideration of the various well-known causes of convulsions, as the predisposing, exciting and proximate. Of the first and second at least, we have learned considerable.

In discussing the causes of disease, before my class, I am accustomed to employ an illustration, which I heard long years ago in the University of Louisville, $\mathrm{K} y$. , and which has seemed to me to be well chosenwhere the different causes generally given as the predisposing, exciting and proximate, or immediate, were compared to making a fire in a stove or grate; in this the combustible material arranged first is the predisposing, the match the exciting, and the union of the oxygen of the air with the carbon of the fuel is the proximate cause of the resultant combustion. I am aware, however, that some object to the designation "Proximate Cause," and I find it much less frequently employed than formerly, as it is really only the ultimate effect, rather than a cause. Nevertheless, I shall hold the illustration as an apt and proper one to enforce an understanding of the operation of the causes of disease.

In considering the predisposing causes, we must note as contributory, age, sex, heredity, temperament, environments, habits, season, diathesis, etc., while the exciting causes, as so well grouped by Rotch and other authors, will include as "Central": 1. Diseases of high temperature. 2. Diseases accompanied by vascular stasis. 3. Diseases characterized by anemia and exhaustion. 4. Various toxic causes. 5. Organic central lesions. 6. Presumably organic disturbances of the brain, as epilepsy, which is excluded from this discussion, and of "Peripheral" (or reflex) causes; rachitis, food, intestinal parasites, dental irritation, foreign bodies (in the ear, nose, etc.), hot baths, burns, etc., mental disturbances (such as fright, etc.) and many others like these; while the "Proximate Causes" must be considered with the points of origin and essential nature of these "Nerve Explosions."

Of all the manifold predisposing causes of convulsions in young children, the most important one is the natural instability of the nervous centers, characteristic of early life, and associated with the non-development of voluntary centers of the cortex; hence it is that age is a most important factor in the etiology of convulsions; and under 2 years is recognized as by far the most susceptible period, for patent reasons already stated. Statistics, indeed, show that over 60 per cent. of deaths from convulsions, up to 20 years, occur in infants under 1 year of age. Convulsions are not only more common in infancy, but much more fatal than later in life, and for reasons that are very apparent. It has been stated by some good observers that males seem to be more susceptible than females; indeed, carefully-gathered statistics seem to justify this conclusion, but it has been suggested by others that inasmuch as more males than females are born each year, the larger number of deaths in males may thus be reconciled, for surely it would be contrary to reasonable expectation, as females are more delicately organized, while the exciting causes are probably about equal. While high atmospheric temperature is a recognized predisposing cause, we also recognize the fact that elevated bodily temperature in infants may be an exciting cause of convulsions, where there is a predisposition to those motor nervous explosions. A temperature, much above $103 \mathrm{~F}$., continuing for several hours, in a highly neurotic child, is 
very likely to induce convulsions. I have seen an attack of convulsions precipitated in a very neurotic infant, from the application of a sharp sinapism, and more than once from a cantharidal vesicant.

I think there is sufficient evidence to justify the conclusion that there often exists in young children, what has been aptly called "a conclusive tendency," probably hereditary, when the predisposition is so marked that very slight excitation, such as anger or fright, or a trivial traumatism, indigestion, coryza, etc., may precipitate a convulsion; and which, in an infant of naturally a more stable nervous equilibrium, would do no harm. Severe burns or scalds are very likely to produce convulsions. One of the most persistent cases of eclampsia I have ever seen occurred recently from a severe scald involving most of the surface of the trunk and abdomen of a girl, 3 years old, who was in almost constant clonic spasms for twenty-four hours before death came to her relief.

Convulsions in infants are not uncommon in the jnception of the graver forms of scarlet fever, and, if after the evolution of the rash, are almost uniformly fatal.

As to what exact rôle dentition plays in the causation of convulsions, good observers are not well agreed; but of the many attacks of eclampsia which $I$ have witnessed in young children during dentition, I can not remember one case where $I$ could be sure that the eruption of the teeth was more than a predisposing cause of the spasm; and I can affirm the same of the so-often accused intestinal worms. These may often be contributory, as one only of several factors, inducing convulsions, and should not be entirely ignored. Jamieson correctly remarks: "Dentition as an exciting cause of convulsions in infants, is not to be considered until all other possible factors are eliminated, as more than double the number of deaths, from convulsions in children occur during the first years of life, and according to statistics, over two-thirds of these occur during the first six months; so that dentition in these cases can not be taken as a cause."

But of all the causative agents in producing convulsions in young children, undoubtedly the food is the most cornmon; probably, at least one-half of all convulsive attacks in infants and young children can be traced to this cause; either food wholly improper in quality, too much in quantity, or too frequently given. So also, bad hygienic conditions often contribute to this accident. Hence the necessity of emphasizing the fact that infant feeding and infant hygiene are the most important subjects in pediatric teaching.

While infants being fed with commercial foods, cows' milk, ete, are the most frequently attacked, I have several times seen the mother's milk precipitate violent convulsions in a healthy infant, within an hour after nursing, and which ceased when taken off the breast, only to return speedily when the breast milk was resumed. Another fruitful source of convulsions is to be found in rachitis and other forms of acute or chronic malnutrition, especially laryngospasms and tetany. So also toxemia, either from microbic agency or from renal affections, is a well-recognized source of infantile convulsions; and aside from all these factors, and many more, as causations of eclampsia, we must also recognize the fact that convulsions are often only "A Mode of Dying," in young children, when occurring late in acute affections, and continuing until life is extinct.

In regard to the diagnosis of eclampsia, it is not difficult as a rule, as we have only to differentiate from epilepsy, which the paroxysms so nearly resemble; the main point of differentiation is to remember that epilepsy is rare in infants under 3 years, the period when eclampsia is most frequent; that epilepsy, unlike eclampsia, is not preceded nor followed by symptoms of acute illness, and has a strong tendency to recur at more or less regular intervals and to become a chronic affection, but notwithstanding the paroxysm or motor nervous explosion, it does not differ in appearance from that of eclampsia; indeed, we must recognize the fact that what seems to be at first true eclampsia does occasionally merge into genuine epilepsy. Perhaps the most distinctive feature in epilepsy, when recognizable, is the peculiar "aura," which immediately precedes the "fit," and which is never present in ordinary eclampsia; but this being only a subjective symptom, it is of little value in diagnosis in infants and children under 4 or 5 years old.

As to the essential pathology of these convulsions, which are only symptomatic of so many different proximate causes, very little light can be obtained by postmortem investigations; the "findings" will be as various as the producing causes.

When death occurs from general convulsions, it will be either from venous stasis in the brain-or effusion or hemorrhage on to that organ-or else by exhaustion from the prolonged attack. In laryngospasm, it may occur by apnea, or suffocation.

The treatment of convulsions is naturally divided into two parts: to relieve the paroxysm as soon as possible; and to endeavor to prevent its recurrence, by removing the cause, or rather by treating the systemic condition of which the convulsion has been only a symptom. But as the exciting cause can not always be readily determined in these emergencies, and as relief of the paroxysm is imperative, it is most fortunate that there are several agents and procedures available to quell the disastrous nervous explosion, and applicable to all cases, until we can investigate the exciting cause.

The first thing generally done is to immerse the child in a warm bath, from five to fifteen minutes, at a temperature of about 96 to $100 \mathrm{~F}$.; or if there is high bodily temperature, about $90 \mathrm{~F}$., while cold water or ice is applied to the head and face; at the same time it will be well to examine the gums, if teething, for possible dental irritation, and if found, which will be only seldom, the gums should be lanced. Next, if good reasons exist for suspecting digestive troubles, and there is no great apparent cerebral congestion, an emetic of ipecac with or without alum; or a hypodermic injection of apomorphia, 1-20 to 1-40 gr. will serve as well. My experience leads me to believe that the dangers of emetics in convulsions, in producing or intensifying cerebral trouble, have been greatly exaggerated, and that they are often indicated, not only as local aids, but as antispasmodics, relaxants, and often calmants and antipyretics, as well as sudorifics and revulsives. If, however, it seems probable that the source of irritation is in the alimentary canal, below the stomach, an enema of warm water with common salt will be in order, and, as soon as possible, a cathartic of calomel, ipecac, and bicarbonate of soda may be given, to be followed in four hours by castor-oil and turpentin-or if in haste, a saline. If the child is in a paroxysm when we enter the chamber, besides the remedies already mentioned, and indeed as a first remedy to "break the spasm," chloroform inhalation will be proper.

After these preliminaries, our next best resources are in the administration of the bromids, and some- 
times chloral, etler per os or per rectum; they may be given separately or combined, when both are indicated, as they often will be. My preference has generally been for sodium or potassium bromid $5 \mathrm{i}$, chloral hydrate $\mathrm{gr}$. iii to $\mathrm{v}$, according to age, given in solutions per rectum. In some well-chosen cases, without cerebral congestion, and when there is great pain, a prompt hypodermic injection of morphia and atropia will yield good results in calming the nervous excitation, but demands great discrimination in its employment, especially in very young infants.

In what smitl and some others call "sympathetic" convulsions, i. e., those occurring from some acute disease, outside the cerebrum or central nervous system, opium in some form is often very useful in quieting the nervous excitement. Happily such heroic measures as venesection, blisters, and severe counterirritation to the surface of the body of infants to "break spasms" have long since become obsolete; nevertheless we occasionally sec cases of convulsions where venesection might serve us as well, and it is a question with some of us older physicians, whether the pendulum in venesection in children has not swung too far in the opposite direction. If there is high temperature, antipyretics and cool sponging, or the "pack" are always indicated. However, after the paroxysms have been controlled, the remedies should be addressed to the removal and prevention of the exciting cause, and later also to the predisposing conditions, as far as possible. This means especial attention to details of diet, hygiene, etc., which so often contribute largely, if not wholly, to the production of these convulsions. The bromids should generally be continued for some time after the paroxysms cease, in order to prevent their recurrence, as well as to remedy the results often left on the nervous centers. It is well known that one convulsion predisposes to another, and sometimes a series ending in epilepsy, which so closely resembles eclampsia in its clinical symptoms and objective features.

If the convulsions are found to be what some of our authors denominate "symptomatic," though always "symptomatic"-but especially of brain lesions-or "sympathetic" of affections other than cerebral disorders, the management will be properly addressed to those pathologic systemic affections, the discussion of which can not come within the limited scope of this paper; and although admitted that laryngospasms of infants are so closely connected with general spasms, I have not deemed it wise to attempt their discussion in this paper.

If it be a fact, and it doubtless is so, that some cases of convulsions require depletion, others repletion, others evacuants only; others antispasmodics and calmants on!y, while still others demand refrigerants and revulsives, how very much discrimination is demanded in the treatment of an affection, which, be it remembered, is always only a symptom-though often a dangerous one - of so many diverse conditions, either temporary-i. e., functional-or permanent (organic) ; also what demand for ready, cool and prompt action.

Impressed, as I have been, with these suggestions, it has been my method to emphasize to my class the importance of always having with them when becoming practitioners, something like an emergency outfit for the prompt management of convulsions, during and between paroxysms. The first and most important of this will be clear, cool, and ready intelligence. Then the armamentarium should include chloroform, bromids, chloral. ipecas, apomorphia, a good hypodermic syringe -in good order-with proper tablets of morphia, atropin, hyoscyamin hydrobromate, a good fountain syringe, and a stomach-tube, fever thermometer and catheter, as it may be in the night and some distance from a drug store, when and where the physician is called in these emergencies; hence the necessity of being always ready for prompt action.

307 Plymouth Avenue.

DIscussion.

Dr. A. C. Cotron, Chicago-The last sentence of this extremely interesting paper aroused in my recollection a series of observations along a line which has particularly interested me during the past five or six years. A year ago, at the meeting of this Section in Denver, I read a paper on the subject of "Disturbed Lactation, Some Causes and Effects." Among some of the effects I called attention to not infrequent attacks of convulsions, apparently as a result of discontinuance of lactation. During the last year I have concentrated my attention somewhat more closely along this line of observation, and among all the causes assigned in the able paper just read for the production of convulsions, it seems to me that food plays the most important part. Errors in diet, arrest of digestion, imperfect assimilation, with the consequent evolution of ptomains which produce intoxication of the nerve-centers, whether by reflexes from chemical irritation in the alimentary tract, or from pressure of gases in the bowel-all excite the nervous system, and cause convulsions; but I believe that dietetic errors and imperfect digestion are the most frequent exciting causes of convulsive seizures. My attention has been called more particularly to convulsive seizures in infants at the breast. There the supposition is that there is not so much liability to dietetic errors as in artificially fed infants, but some careful observations on that point will satisfy you, I think, that very frequently the quality of the milk secreted in otherwise normal mammary glands is subject to sudden and very positive changes, not only in the grosser aspects, but in its chemical reactions. Mental influences, hygienic influences, climatic, thermic and many other influences will induce a change in the quality of the mother's milk. From the recorded observations it would appear that the most pronounced causes are those which affect the emotions-the psychic influences. In my paper of a year ago I alluded to the effect of poisons on a nursing woman. In at least seventy-two cases, the opportunities for observation were especially favorable, and these received my most careful attention. I reported fifty-one cases one year ago. Since then my attention has been called to a number more. Careful scrutiny, I think, will show that not infrequently undue, unusual, or excessive ccitus on the part of the nursing woman will suddenly and remarkably affect the quality of the milk, and produce a marked disturbance in the nursling, which is very commonly manifested by convulsions, and occasionally, even by death.

Dr. Krmball of Indiana-The etiologic factors which enter into these cases are perhaps generally understood by the profession, but how to manage such cases is a problem of much interest, at least to me. Of all the exciting causes, I have found indigestion, or the toxins arising in the alimentary tract the most common. I recall one case in which there was not only the usual convulsion, but the forearms were flexed on the arms and the hands on the forearms, and assoclated with this was a peculiar distortion of the face. Although the mother said that the child had not eaten anything out of the way, I administered a calomel purge. The next day, on visiting the patient, a child of 4 years, it was looking considerably better. The mother then told me that the child had evacuated nearly a tea-cup of beans. I recall another case in which every time the child ate peanuts it was necessary to call on me. In the treatment of these cases I have derived especial satisfaction from the administration of gelsemium. Its action is, of course, on the cerebrospinal centers. It seems to control the convulsion more quickly than anything else $I$ have used, and it is easily given.

Dr. CotToN-Don't you think the calomel purge is better than the gelsemium?

Dr. Kimball-Yes, but I have often found that while the calomel was acting the gelsemium proved useful by calming the cerebrospinal system.

Dr. EwING, Salt Lake City, Utah-Aside from the causes which have been enumerated, and especially the overfeeding of children, I find, in male subjects, that a most frequent cause of spasms is an adherent prepuce. I usually examine the prepuce in cases of convulsions, and I have found that by remedying this many cures could be affected. It is my custom to wash out the bowel with abrut half a gallon of water, and also 
to give a calomel purge instead of simply treating the fever.

DR. J. A. Work, Elkhart, Ind-Every clfect has its cause, and hence when convulsions are present we should look for the cause of the pathologic condition. I believe if we are careful we can usually ascertain the cause, and then it is a comparatively easy matter to remove it. I remember, about twenty years ago, treating a very severe case of convulsions in a child about ten months old. The next day we found in the stools a small piece of corn-cob, and the nexit day a small piece of fish.

Di. Foster of Illinois-It seems to me that in the enumeration of these etiologic factors we should go just a little further and try to find out whether there is a stimulation of the nervecells of the brain, especially the motor areas, or whether there is a depression of these areas. What is the pathologic process which is taking place in the nerve-cells of the brain? That is what we wish to know. Some physicians give stimulants; others give bromids with chloral. To determine which treatment is proper we should know what is the process to which these nervecells are subjected. There is a clonic convulsion present. In the majority of cases, I think, instead of the nerve-cells being stimulated they are depressed, and their normal tone lowered. This indicates the line of treatment to be followed.

Dr. E. E. MAYkl, Pittsburg, Pa.-This reminds me of Fleschig's theory of the association fibers of the brain, which he claims govern the projection fibers and thus exercise an inhibitory influence on the motor zones. In children these fibers are $110 \mathrm{t}$ developed, and hence the cortical cells in childhood are easily excited. Whether his hypothesis is correct, I can not say, but it is certain that the inhibitory power of a child's brain is much less than that of an adult. Nothnagel's epileptic center in the pons may also aid in the production of convulsions.

One fact must be remembered before everything else. (oulvulsions are only a symptom. No diagnosis is made by saying a child has convulsions, any more than if you say a child has pain. What causes the convulsions. to what disease is it due? What has excited the cortical irritation?

All the previous speakers have spoken of the convulsions due to reflex causes, to poisons in the blood, to infection etc., but no one has emphasized convulsions due to organic brain disease. Convulsions are of.ten the first symptom of brain disease, and, unitil other pronounced symptoms are fortheoming, the physician does not think of brain tumor, brain abscess, ete. A convujsion is also often the first sign of a future epilepsy.

Treatment then, can only be successful if the cause is founl and the excitiag factor removed. To check the convulsion rive chloroform, and then at my leisure search for the cause and treat it. Wn matter what the cause is, bromids in arses of 2 to 5 grains are advisable to allay cortical irritation.

Dr. Foster of Illinois-Will not the same stimulation in the nerve-cells of the child produce the same result as in the nerve-cells of the adult brain?

Dr. Rosa ENgELMan-From the very fact that anatomic studies of the brain of the child show lack of development it is easy to account for all forms of convulsions, whether toxic, refiex or what not.

Dr. C. G. Slagle, Minneapolis, Minn.-This is a large subject, and one which can not be satisfactorily treated in one paper, but I am glad that it has elicited such a full discussion, and I thank you for it. I hope Dr. Cotton will pursue his in teresting investigations further. I am sorry that I did not have time to discuss the treatment fully. To check the spasm, the best method is the inhalation of chloroform. It is also useful to give an enema of salt water, botl to produce an evacuation of the bowel, and because of its revulsive effect. It is also desirable in many cases to give an emetic, particularly in those cases in which from the history it seems probable that the undigested food has not passed beyond the stomach. think I get just as good results from enemas of salt water as from enemas containing bromids. The adherent prepuce is certainly a contributory cause, but $I$ doubt if it is an exciting cause. It is true that we must search for the cause, but for the immediate needs we can satisfactorily control the convulsion with chloroform as I have said.

\section{Lanolin in Reduction of Enlarged Glands.}

A. C. Frickenhaus (Monatshefte fur Prakt. Dermatol.) reports the rapid reduction in size of enlarged glands after in unction with lanolin. The axillary glands were enlarged and painful, following recurring furunculosis of the trunk; and thorough application of lanolin over the enlarged glands was followed by diminution in their size and marked lessening of pain. Similar results were obtained in a case of angina tonsillaris, accompanied by enlargement of the tonsils and pain on swallowing.

\section{CONCERNING THE COMPLICATIONS OF ACUTE OTITIS MEDIA PURULENTA.* \\ BY E. S. ALLEN, M.D. \\ CINCINNATY, OHIO.}

The subject is given as above out of deference to the ordinary dictum. It would be more correct to use the expression: "Serious and fatal forms of acute otitis," rather than "complications." The attempt will be made to show that we have to do with a distinct variety of ctitis rather than with complications following the ordinary middle-ear suppurations, that dangerous and fatal meningeal trouble in connection with acute middleear suppuration represents a distinct type of infective inflammation. Acute otitis media is caused by germ infection reaching the tympanic cavis $y$, in nearly all cases through the Eustachian tube. Infection by means of the blood and lymph vessels is possible, but difficult to prove. Auto-infection probably does not take place, as under normal conditions the middle ear is probably free from germs. The character of the resulting inflammation depends on the relation between two factors-the virulence of the organisms and the resistance of the tissues, the same organisms producing in one case a virulent suppuration and in others but a purely serous or mucous exudation. At an early stage, i. e., before perforation, it is generally impossible to diagnose between a severe catarrhal inflammation and an acute suppuration. In acute exudative otitis media we generally see a bulging of the drum membrane, indicating that the secretion is accumulating under pressure. This can be only, and it is extremely pertinent to the subject under consideration, because the swelling of the mucous membrane has shut off the entrance into attic or antrum and confined the exudate to the tympanic cavity proper. Secretion accumulating in the middle ear under pressure is generally non-purulent or but slightly purulent, the pressure preventing the exudation of leucocytes. This we know from the fact that nearly always when we make a paracentesis the exudate that first appears is not purulent and that a certain time elapses before free suppuration sets in. The suppuration coming on in a few hours or next day is not due to the infection of the cavity after paracentesis, but is due to the relief of the pressure. We can easily open the drum cavity without inrecting it. A similar occurrence takes place probably when perforation occurs spontaneously. The acute cases may end in one of four ways:

1. Fverything being favorable, and no secondary infection taking place, the suppuration gradually ceases and recovery occurs.

2. In neglected cases a chronic otorrhea is established.

3. At other times after a varying period, generally while the suppuration is still profuse, the mastoid cells become involved in a pronounced way.

4. On other and not too seldom occasions we get no decisive mastoid or other symptoms, but at a time varying from a few days to several months from the onset of the ear symptoms, meningitis, pyemia, and symptoms of thrombosis or abscess set in.

I have purposely separated 3 and 4 because while brain symptoms may arise in neglected cases of mastoid involvement, still, ordinarily brain symptoms occur without the general free mastoid suppuration and necrosis which we see in ordinary mastoiditis. It is this fourth variety of acute inflammatory ear trouble which $I$ desire to discuss. Can these serious or fatal conditions be

* Presented to the Section on Laryngology and otology, at the Fiftieth Annual Meeting of the American Medical Association. held at Columbus, Ohio, June 6-9, 1899. 